\title{
ON DIFFERENCES IN DIFFERENTIAL ROTATION
}

\author{
W. VAN TEND and C. ZWAAN \\ Astronomical Institute, Utrecht, The Netherlands
}

Summary. We investigated the reality of the differences in rotation rates found from various features. We only used published observations including almost forgotten work of more than half a century ago.

Here we summarize some conclusions which seem interesting in the present context. A more detailed account will be submitted to Solar Physics where also the references will be given.

(1) It is well known that larger rotation rates are found from sunspots and faculae than from photospheric Doppler shifts. However, the faculae and the rare spots occurring outside the activity belt indicate that the differences diminish for latitudes $|\phi|>40^{\circ}$ (see Figure 1).

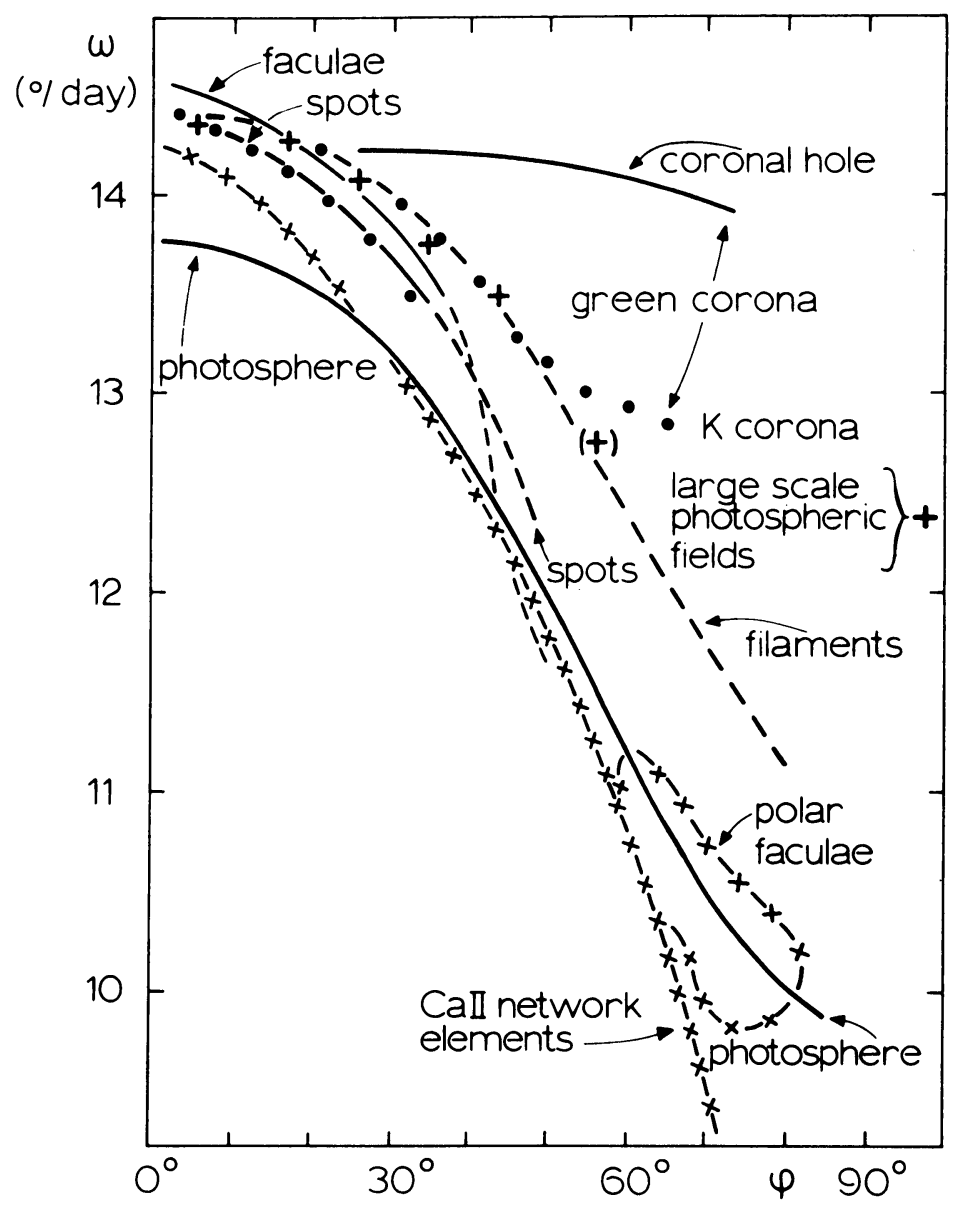

Fig. 1. Mean sidereal rotation rates $\omega$ as a function of heliographic latitude $\phi$. 
(2) There are no significant differences between the rotation rates determined from photospheric Doppler shifts, from white-light polar faculae, and from Ca II K 3 network elements outside active regions (Milošević), at least for latitudes $|\phi|>20^{\circ}$. In other words, magnetic flux tubes not (or, no longer?) belonging to active regions corotate with the photospheric plasma.

(3) There is no difference between the rotation rates derived from filaments and from autocorrelation analysis of long series of magnetograph data. This is not surprising since autocorrelation analysis yields mean rotation rates for large magnetic complexes of long duration (many months), whereas filaments mark the boundaries between magnetic complexes of opposite polarity.

(4) However, the intriguing result is that, at least for latitudes $|\phi|>20^{\circ}$, the large complexes rotate faster than all known elements which constitute those complexes: sunspots, faculae and network elements.

(5) Autocorrelation analyses of coronal features yield rotation rates larger than the rotation rates for large photospheric magnetic complexes. More specifically, the $\mathrm{K}$ corona and the short-lived condensations observed in the green line rotate about equally fast or slightly faster than the photospheric complexes whereas the very long-lived quiescent condensations rotate about as fast as the almost rigidly rotating coronal holes.

\section{DISCUSSION}

Howard: I wish to point out that you and other investigators have shown the results, that Harvey and I obtained some years ago in comparison with other rotation data. Often our results are shown as a solid line as if they were well established and invariant. In fact, they are neither. In particular the average rotation rate has been increasing in recent years, and is now early up to the sunspot rate.

Antonucci: At high altitudes the rotation period of the green corona appears to be dependent on the solar cycle phase, in the last two solar cycles. Before solar minimum the rotation period at high latitudes approaches the equatorial one (before 1954 the rotation period was exactly the same at $40^{\circ}-60^{\circ}$ and at $\left.20^{\circ}-0^{\circ}\right)$, near activity maximum the differential rotation curves of the green corona and of the photospheric magnetic fields are in fairly good agreement. Therefore, the behaviour of the green corona could be better described by two different rotation curves corresponding to the period before solar minimum and to solar maximum.

Zwaan: I agree, your results are included in our graph. 